Tovell, VE; Massie, I; Kureshi, AK; Daniels, JT; (2015) Functional Limbal Epithelial Cells Can Be Successfully Isolated From Organ Culture Rims Following Long-Term Storage. Investigative Opthalmology \& Visual Science , 56 (6) 3531 - 3531. 10.1167/iovs.1415429.

\title{
Article
}

\section{Functional limbal epithelial cells can be successfully isolated from organ culture rims following long-term storage}

Running Title: hLEC Isolation from Organ Culture Rims

Victoria E. Tovell, Isobel Massie, Alvena K. Kureshi and Julie T. Daniels.

Department of Ocular Biology and Therapeutics, UCL Institute of Ophthalmology and Moorfields Eye Hospital, 11-43 Bath Street, London, EC1V 9EL, UK.

\section{Corresponding author:}

Victoria Tovell

Department of Ocular Biology and Therapeutics

UCL Institute of Ophthalmology,

11-43 Bath Street, London, EC1V 9EL, UK.

Tel: +44 (0) 76086996

Fax: +44 (0) 76086887

Email: v.tovell@ucl.ac.uk

Word count abstract: 245

Word count text: 3516 


\section{ABSTRACT}

\section{PURPOSE:}

Due to a shortage of fresh corneal tissue for research, it was of interest to investigate the potential of successfully isolating human limbal epithelial cells (hLECs) from organ culture corneal-scleral (OCCS) rims.

METHODS:

Superficial segments of corneal limbus were dissected and digested using collagenase $\left(0.5 \mathrm{mg} / \mathrm{ml}, 16 \mathrm{hrs}\right.$ at $\left.37^{\circ} \mathrm{C}\right)$. Cell suspensions were separated into four different growth conditions (Corneal epithelial cell media, CM; CM + 3T3; Stromal stem cell media, SM; and $\mathrm{SM}+3 \mathrm{~T} 3)$. Colony number, hLEC count, cell density and colony forming efficiency (CFE) were quantified to assess different growth conditions. The expression profile associated with basal hLECs was assessed by immunofluorescence and epithelial integrity was measured using our RAFT corneal tissue equivalent.

RESULTS:

hLECs can be successfully isolated from OCCS rims following 4 weeks in storage with an $80.55 \%$ success rate out of 36 corneal rims. SM +3 T3s provided optimal growth conditions. Colony number, total cell number and cell density were significantly higher at day 7 in cultures with SM compared to CM. There were no significant differences found between SM and CM when assessing CFE and the expression profile associated with basal hLECs. Cells maintained in SM compared to CM were found to produce a higher quality epithelium.

CONCLUSION:

OCCS rims can be a valuable source for hLEC. Using a combination of collagenase-based isolation and media designed for stromal stem cell isolation, a high number of good quality hLECs can be cultured from tissue that would have otherwise been ignored.

\section{INTRODUCTION}

Corneal maintenance and transparency are essential for normal vision and are facilitated by the continuous renewal of the corneal epithelium. The superficial cornea is susceptible to a number of insults and injuries that can damage the limbal epithelial stem cells (LESCs) responsible for this physiological renewal process. The continuous importance of isolating LESCs for research into stem cell therapies begs for more detailed studies into optimal isolation techniques. However, a worldwide shortage in donor corneas for transplantation reflects a shortage of corneal-scleral rims available to the research community. This problem is exacerbated due to different storage methods of corneas for transplantation, ${ }^{1,2}$ with the preferred short-term storage method for cell isolation being less prevalent in the majority of European eye banks. ${ }^{3}$

The two main methods of corneal storage adopted by European eye banks are organ culture and hypothermic storage. Organ culture involves long term (3-4 weeks) storage in culture medium supplemented with fetal calf serum, antibiotics and antimycotics at room temperature to $37^{\circ} \mathrm{C}$, while hypothermic storage involves short term (up to 7 days) maintenance in commercially available media such as Optisol-GS at $2-8^{\circ} \mathrm{C} .^{2}$ Although hypothermic storage is the most popular storage method worldwide, the majority of European Eye Banks tend to opt for Organ culture Storage due to the extended storage time. ${ }^{3}$ Since 2009, the number of organ culture stored corneas processed at the Moorfields Lions Eye Bank (London, UK) was 2.5 fold higher than hypothermic stored corneas. ${ }^{4}$ 
A major clinical requirement of corneal transplantation is a healthy endothelium. Organ culture preservation of corneas for transplantation was therefore introduced as a way to monitor the stability of the endothelium during storage, which provides the knowledge to eliminate any corneas that may not be successful for transplantation. ${ }^{5-7}$ There are also a number of advantages of long term donor tissue storage prior to surgery such as offering more possibilities in terms of operation schedules, tissue type matching and also minimizing the waste of donor tissue, all of which are more restricted with hypothermic storage due to time constraints. ${ }^{2}$

The research community tends to prefer short-term stored or fresh tissue for human limbal epithelial cell (hLEC) and LESC isolation, both for research and stem cell therapy purposes. However, a few studies have indicated a potential for using organ culture corneal-scleral (OCCS) rims to isolate limbal epithelial cells, suggesting a possible use for OCCS rims in hLEC isolation ${ }^{8,9}$ and even limbal allo-graft transplantation. ${ }^{10,11}$ All of these studies have focused on the limbal explant as a method for hLEC isolation. However emerging evidence also suggests a collagenase-based method as promising tool for hLEC isolation and expansion. ${ }^{12,13}$ Chen and colleagues first demonstrated the advantages of using collagenase to release basal progenitor cells from the limbus of fresh tissue in a culture method that does not rely on $3 \mathrm{~T} 3 \mathrm{~s} .{ }^{12}$ In this study we investigate the use collagenase digestion as a method to isolate hLECs from OCCS rims. We also assess the effects of different media and the presence of a 3T3 feeder layer on the success of these cultures with the aim of introducing a robust and reproducible method of hLEC isolation from organ culture rims.

\section{METHODS}

\section{Materials}

Cadaveric donor corneal-scleral rims with appropriate research consent were obtained from Moorfields Lions Eye Bank (London, UK). Ethical permission for this study was obtained from the Research Ethics Committee (UK, ref. no. 10/H0106/57-11ETR10) and all tissue was handled in accordance with the Declaration of Helsinki. Corneas were stored by organ culture at ambient temperature after enucleation for 4-6 weeks before hLEC isolation.

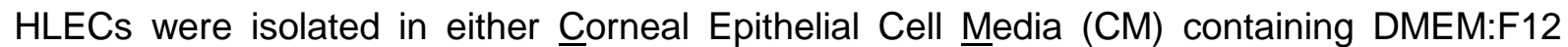
basal medium (3:1), 10\% fetal bovine serum, 1\% antibiotic-antimycotic, Epidermal Growth Factor (EGF) $(10 \mathrm{ng} / \mathrm{ml}$; Life Technologies Ltd, Paisley, UK), hydrocortisone $(0.4 \mu \mathrm{g} / \mathrm{ml})$, insulin $(5 \mu \mathrm{g} / \mathrm{ml})$, adenine $(0.18 \mathrm{mM})$, transferrin $(5 \mu \mathrm{g} / \mathrm{ml})$, T3 $(2 \mathrm{nM})$, cholera toxin $(0.1 \mathrm{nM}$; Sigma-Aldrich, Dorset, UK) or Stromal Stem Cell Media (SM) containing DMEM:MCDB-201 (3:2, Sigma-Aldrich), $2 \%$ fetal bovine serum, penicillin $(100 \mathrm{lU} / \mathrm{ml})$, streptomycin $(100 \mu \mathrm{g} / \mathrm{ml})$, gentamycin $(50 \mu \mathrm{g} / \mathrm{ml})$, insulin $(10 \mathrm{mg} / \mathrm{ml})$ - transferrin $(5.5 \mathrm{mg} / \mathrm{ml})$ - selenous acid $(6.7 \mathrm{ng} / \mathrm{ml})$, (ITS, 1X, Life Technologies), albuMAX-I (1 mg/ml), Dexamethasone (10nM), EGF (10 $\mathrm{ng} / \mathrm{ml})$, L-Ascorbic acid 2-phosphate $(120 \mu \mathrm{M})$, cholera toxin $(100 \mathrm{ng} / \mathrm{ml})$, platelet-derived growth factor $(10 \mathrm{ng} / \mathrm{ml}$; Sigma-Aldrich). Isolation of hLECs was carried out with or without a feeder layer $(F)$ of 3 T3-Swiss albino cells $\left(\mathrm{ATCC}^{\circledR} \mathrm{CCL}^{\circledR}-92^{\mathrm{TM}}\right)$, which were growth arrested with $4 \mu \mathrm{g} / \mathrm{ml}$ mitomycin $\mathrm{C}$ (Sigma-Aldrich) for $2 \mathrm{~h}$ at $37^{\circ} \mathrm{C}$. 


\section{Isolation and Culture of hLECs}

Isolation of hLECs was carried out using the same isolation method that is used for corneal stromal stem cell (CSSC) isolation. ${ }^{14,15}$ For this, a thin layer of superficial limbus containing the limbal crypts was trimmed away from the remaining stroma of OCCS rims (Fig. 1A) using fine sprung scissors. The superficial limbus was cut into $2 \mathrm{~mm}$ segments with a scalpel (Fig. 1B) before incubating with $0.5 \mathrm{mg} / \mathrm{ml}$ collagenase type-L (Sigma-Aldrich) for 16 hours at $37^{\circ} \mathrm{C}$. Cells and tissue were dissociated by pipetting up and down with a P1000 and the cell pellet was collected by centrifugation before resuspending in the desired media (CM or SM). Mixed populations of epithelial cells/clusters and stromal cells were cultured in 6 well plates unless otherwise stated, with or without a feeder layer. All cultures were incubated at $37^{\circ} \mathrm{C}$ with 5\% CO2 in air and media was changed three times a week.

For direct comparison of the dispase and collagenase isolation methods, OCCS rims were bisected into temporal and nasal segments in which one half was treated with collagenase (as above) and the other half with dispase. Dispase segments were incubated overnight in $1.2 \mathrm{U} / \mathrm{ml}$ dispase (Roche Pharmaceuticals, Welwyn Garden City, UK) at $4^{\circ} \mathrm{C}$ before hLECs were mechanically scraped into fresh $\mathrm{CM}$ using forceps to generate a cell suspension.

\section{Rhodamine Staining}

For Rhodamine staining, cultures were fixed at day 7 or 14 in $4 \%$ paraformaldehyde (PFA; VWR, Soulbury, UK) for 10 minutes at room temperature before staining with $1 \%$ Rhodamine B (Sigma-Aldrich) for 10 minutes at room temperature. Cells were then gently rinsed in water five times and left inverted overnight to air-dry. Images were captured using a digital camera and light box.

\section{Cell Density, Colony Number and Total hLEC Number}

OCCS rims were digested using collagenase and each cell suspension was divided between the four different growth conditions. At day seven, three sets of photomicrographs were captured at random for each cell culture condition. The total number of cells in each field of view was counted using ImageJ software and data expressed as cell number $/ \mathrm{mm}^{2}$. Cultures were also analysed for total colony number by counting the number of colonies at day 7 by viewing under a microscope. Aborted colonies were also counted and identified as small, highly irregular and terminal colonies as originally described by Barrandon and Green. ${ }^{16}$ Total hLEC number was also counted and analysed. Cells were trypsinised using $0.5 \%$ trypsin-EDTA (Life Technologies), after initial removal of 3T3 cells with $0.05 \%$ trypsin-EDTA, and counted using a hemocytometer.

\section{Colony Forming Efficiency}

OCCS rims were digested using collagenase and each cell suspension was divided between the four different growth conditions. At day seven, hLECs were trypsinised and counted as above. 750 cells from each growth condition were seeded onto new growth-arrested 3T3 feeder layers in 6 well plates and cultured for 7-10 days. Cells were fixed and stained with Rhodamine $B$, images were captured using a light box and colonies were counted using ImageJ software. Colony forming efficiency (CFE) was expressed as a percentage of the number of colonies per cell plated using the following equation: 


$$
\text { CFE }(\%)=\frac{\text { number of colonies }}{\text { number of cells seeded }} \times 100
$$

\section{Statistical Analysis}

Data are represented as mean \pm standard error of the mean (SEM) for a minimum of 5 OCCS rims. Statistical differences were calculated in excel using Students unpaired t-test to compare 2 groups, $\mathrm{CM}: \mathrm{CM}(\mathrm{F})$ and $\mathrm{SM}: \mathrm{SM}(\mathrm{F}), \mathrm{CM}: \mathrm{SM}$ and $\mathrm{CM}(\mathrm{F}): \mathrm{SM}(\mathrm{F}) . \quad \mathrm{p}<0.05$ was considered statistically significant.

\section{RAFT Tissue Equivalent}

RAFT tissue equivalents (TEs) were made by preparing CSSC populated collagen matrices using $80 \%$ v/v type I rat-tail collagen solution ( $2 \mathrm{mg} / \mathrm{ml}$; First Link, Birmingham, UK) and $10 \%$ $\mathrm{vol} / \mathrm{vol}$ 10X Minimum Essential Medium (Life Technologies). 10\% v/v CSSCs in SM were added after neutralization at a density of 1 million cells $/ \mathrm{ml}$. A volume of $2.2 \mathrm{ml}$ of gel solution was cast into the wells of a 12 well plate and allowed to set for 30 minutes at $37^{\circ} \mathrm{C}$. Confined gel compression was carried out as previously described ${ }^{17}$ and the resulting tissue equivalents were submerged in $2 \mathrm{ml}$ of CM or SM containing 1 million hLECs (pre-expanded with 3 T3 feeder cells and SM for 14 days). At day seven, RAFT TEs were transferred epithelial side up onto transwell inserts (Millipore, Watford, UK), and placed in 6 well plates. The bottom chamber was filled with $1 \mathrm{ml}$ media (CM or SM) and media was changed every other day for a week.

\section{Immunofluorescence}

Cells were fixed using 4\% PFA for 10 minutes (cells on plastic) or 30 minutes (cells on RAFT/Epithelial clusters) at room temperature. Samples were washed ( $3 \times 5 \mathrm{~min})$ with DPBS followed by a 10 minute (cells on plastic) or 30 minute (cells on RAFT/Epithelial clusters) incubation in $0.5 \%$ triton DPBS (DPBS-T). Non-specific binding sites were blocked with $5 \%$ goat serum in DBPS-T for 1 hour at room temperature. Samples were incubated over night at $4^{\circ} \mathrm{C}$ in primary antibodies (p63- $\alpha, 1: 100$, Cell Signaling Technology; Cytokeratin 3, mouse anti-keratin K3/K67 monoclonal antibody, 1:200, Millipore; Pax6, Pax-6 polyclonal antibody, 1:100; Vimentin, Anti-Vimentin antibody [SP20], 1:100; PCK, Anti-pan cytokeratin [AE1/AE3], 1:50, Abcam) diluted in 1\% goat serum DPBS-T. Samples were washed and incubated in secondary antibody Alexa Fluor 594 (1:1000; Life Technologies) with PhalloidinTetramethylrhodamine B isothiocyanate (TRITC, $50 \mu \mathrm{g} / \mathrm{ml}$; Sigma-Aldrich) for 1 hour at room temperature. Cells were washed and mounted on slides with vectorshield mounting medium containing 4', 6-diamidino-2-phenylindole (DAPI; Vector Labs, Peterborough, UK) and sealed with nail varnish for imaging on a Zeiss LSM 710 confocal microscope using Zen software. 


\section{RESULTS}

\section{CSSC isolation technique is a useful tool for hLEC isolation}

A technique used to isolate CSSCs ${ }^{15}$ was also found to be a valuable method for hLEC isolation. By digesting the superficial layer of the limbus (Fig. 1A-B) in which limbal crypts are evident (Fig. 1C), cell suspensions containing a mixed population of limbal epithelial cells/clusters (Fig. $1 \mathrm{D}-\mathrm{E}$ ) and limbal stromal cells (amongst other undefined cell types) were generated. Colonies of hLECs were evident after 24 hours in culture (Fig. 1F) and continued to expand with stomal cells lining the edges of colonies (Fig. 1G). After 7-14 days in culture, hLEC colonies maintained a corneal epithelial phenotype (Fig. $1 \mathrm{H}$ ). Before stromal cells became confluent, CSSCs could be selectively trypsinised to produce pure CSSC cultures from the same OCCS rim (Fig. 1I).

Limbal epithelial clusters isolated using collagenase digestion expressed both vimentin and pan-cytokeratin (PCK) suggesting the presence of both mesenchymal cells as well as epithelial cells (Fig. 2). It is clear to see the advantage of using collagenase over dispase to isolate hLECs from OCCS rims when directly comparing these isolation methods (Fig. 3A). This is in agreement with previously reported data comparing dispase and collagenase isolation methods. ${ }^{12}$ After 14 days in culture, using the collagenase isolation method, hLECs covered over $50 \%$ of a $55 \mathrm{~mm}^{2}$ culture dish (Fig. 3B, top). This was reproducible in most samples with $80.55 \%$ of 36 organ culture corneal-scleral rims producing epithelial colonies and the remaining $19.45 \%$ of samples failing to produce epithelial colonies but instead producing a confluent layer of stromal cells (Fig. 3B, bottom). No correlation was found between successful cultures and donor characteristics.

\section{Effects of 3T3 feeders and SM on hLEC isolation and culture}

Due to the observations of successful hLEC isolation from long-term stored OCCS rims, it was important to investigate the difference between SM (designed for isolation CSSCs) ${ }^{14}$ and $\mathrm{CM}$ (the media we routinely use for hLEC isolation). Following collagenase digestion of OCCS rims, each cell suspension was separated into four different growth conditions; 1) CM only, 2) CM + 3T3 feeders, 3) SM only, 4) SM + 3T3 feeders. Figure 4 shows that although initial hLEC colonies were slightly larger in CM and SM without 3T3 feeders at day 1 (top panel), after 7 days in culture these differences were eliminated (middle panel). Colonies cultured in SM, either with or without 3T3 feeders, were more successful and more organized than those cultured in CM, with or without 3T3 feeders. Furthermore, rhodamine staining at day 14 (bottom panel) showed a high number of aborted colonies in CM compared to SM growth conditions, suggesting that SM is preferable for hLEC isolation.

Analysis of cell growth was also carried out for these four conditions. Figure 5A shows the total number of colonies counted for each growth condition at day 7 of culture. A significantly higher number (Student's t-test; $p<0.05$ ) of total colonies were counted in CM with 3T3 feeders $(\mathrm{CM}(\mathrm{F}))$ compared to $\mathrm{CM}$ alone, and in SM with feeders $(\mathrm{SM}(\mathrm{F}))$ compared to $\mathrm{SM}$ alone. A significantly higher number (Student's t-test; $p<0.05$ ) of total colonies were also counted in $\mathrm{CM}(\mathrm{F})$ cultures compared to $\mathrm{SM}(\mathrm{F})$ cultures. Although there was no significant difference in total colony counts found between $\mathrm{CM}(\mathrm{F})$ and $\mathrm{SM}(\mathrm{F})$, when aborted colony number was counted there were significantly more aborted colonies in $\mathrm{CM}(\mathrm{F})$ cultures compared to $\mathrm{SM}(\mathrm{F})$ cultures (Fig. 5B). This was reflected in total hLEC count in that there were significantly more $h L E C$ s counted in $S M(F)$ cultures compared to $\mathrm{CM}(\mathrm{F})$ cultures (Fig. $5 \mathrm{C})$. There was also a significantly higher number of hLECs in SM cultured compared to CM 
cultures, and in $\mathrm{CM}(\mathrm{F})$ cultured compared to $\mathrm{CM}$ cultures. Overall these data suggest a more successful hLEC isolation in SM compared to CM and that hLEC isolation favours the presence of 3T3 feeder cells. A summary of the percentage of successful hLEC isolations from OCCS rims in this particular experiment is represented in Figure 5D.

\section{hLECs isolated from OCCS rims are a potential source of LESCs}

Cell size was analysed by counting confluent patches of cells to give an overall cell density. Cells on three separate photomicrographs, per condition per $n$ number, were counted using imageJ software. Figure 6 shows the different cell densities produced using different growth conditions. SM yields higher cell densities per unit area than $\mathrm{CM}$ indicating the maintenance of small, poorly differentiated hLECs (Fig. 6A and C). However, no significant differences were found between these four conditions when assessing the colony forming efficiency of the cultures (Fig. 6B and D).

Protein expression associated with limbal basal epithelial cells (p63 $\alpha$ ) and terminally differentiating suprabasal cells (CK3) were assessed in cells isolated with 3T3 feeders in the presence of either CM or SM (Fig. 7). Positive expression for $p 63 \alpha$ and a negative expression of cytokeratin 3 (CK3) corresponded to poorly differentiated hLEC profile, with the exception of a small amount of CK3 staining indicating the presence of larger more differentiated epithelial cells. Pax6 was used as a corneal epithelial cell marker and was positively expressed in hLECs maintained in CM and SM.

\section{hLECs grown in SM support a corneal phenotype}

Pre-expanded hLECs that were isolated using SM and 3T3 feeders were seeded onto RAFT TEs to assess their potential to form a multi-layered epithelium. Interestingly, even though hLECs had been pre-expanded in ideal conditions (SM + 3T3s), morphology and epithelial multi-layering was found to differ between RAFT TEs maintained in SM and CM (Fig. 8). Whilst RAFT TEs maintained in SM displayed a typical corneal phenotype, with columnar basal epithelial cells and overlying squamous epithelial cells. RAFT TEs maintained in CM displayed a multi-layering phenotype but lacked structure in terms of basal columnar cells and superficial squamous cells. Differences also became apparent when observing the basal epithelial layer of each RAFT TE (Fig. 8). RAFT TEs maintained in SM presented a tight layer of basal cells with highly organized actin boarders compared to the irregular and differentiated basal cells observed in RAFT TEs maintained in CM. Epithelial cells on RAFT TEs maintained in SM also display a poorly differentiated hLEC profile when observing $p 63 \alpha$, CK3 and Pax6 expression patterns (Fig. 9).

\section{DISCUSSION}

Availability of donor corneal-scleral rims is essential for the progression of basic research in the cornea and research into stem cell therapies for the treatment of corneal disease. Naturally, there is a preference for fresh corneal tissue in the research community since longterm tissue storage has been shown to have a negative impact, for example, on the success and speed of hLEC isolation. ${ }^{18,19}$ However, now that organ culture has become the corneal storage method of choice in most European eye banks, not only have supplies of fresher tissue for research become limited, but also surplus OCCS rims that could be used for research are being overlooked. Hence, it was advantageous to attempt to optimise hLEC 
isolation from OCCS rims. In this study, we demonstrate that functional hLECs can be isolated successfully, and reproducibly cultured, from OCCS rims by using collagenase to digest the superficial limbus.

Several studies have shown that hLECs can be cultured from OCCS rims using tissue explants $^{8-11,18-22}$ or by isolation of a single cell suspension using a combination of dispase and trypsin. ${ }^{9,19-21}$ A direct comparison between cell suspension and explant culture of limbal epithelial cells showed that the former generated a significantly higher number of stem cells compared to the explant technique. ${ }^{20,21}$ In these reported studies dispase was used to generate the epithelial cell suspension. However, recent studies by Chen and colleagues have shown that using collagenase to generate cell suspensions is a valuable technique for isolating LESCs. ${ }^{12}$ This study shows that using dispase and trypsin for LESC isolation separates the basal progenitor cells from their supporting niche cells leading to less successful isolations. Therefore, collagenase isolation may have potential to supersede both the explant method and the dispase/trypsin method of isolation in terms of producing a reliable source of LESCs. Our findings demonstrate that by adapting the collagenase based method used for CSSC isolation ${ }^{14,15}$ and shifting the focus to hLEC isolation; functional hLECs can be successfully and reproducibly isolated from OCCS rims that have been stored for 4 weeks or more.

Interestingly, when isolating hLECs in media that was initially designed for mesenchymal stem cells, ${ }^{23}$ and adapted for CSSC isolation, ${ }^{14}$ hLEC colonies thrive and cultures contain a higher number of colonies compared with those maintained in CM. Studies have shown that different media can have a different effect on LESC growth, cell phenotype and expression of putative LESC markers. ${ }^{24}$ These differences can be due to a number of different factors as there are many components to cell culture media. One striking difference between SM and CM is the concentrations of FBS, $2 \%$ and $10 \%$ respectively. Whilst studies suggest that the FBS stimulates limbal epithelial cell proliferation, ${ }^{25}$ other studies have shown that it is possible to cultivate limbal epithelial stem cells without the use of serum in the media. ${ }^{26,27}$ Our findings suggest that a lower concentration of FBS may be beneficial for hLEC growth as colonies are more likely to become aborted, and cells also become more differentiated when maintained in CM. However, this may not be due to serum content alone as there are other factors involved. Further analysis into the components of these media will need to be carried out to elucidate the contributing factors for successful isolation.

All components involved in hLEC isolation if used for transplantation require consideration in terms of safety and good manufacturing practice (GMP). Although there are banked 3T3s that comply with GMP standards at the National Institute of Biological Standards and Controls, it would be ideal to eliminate the use of animal derived products for use in clinic due to the potential risk of transmitting xenotic adventitious agents. Studies have therefore reported the use of human equivalent feeder layers ${ }^{28,29}$ and indeed culture methods without feeder layers. ${ }^{12}$ Similar to Chen and colleagues, ${ }^{12}$ we have found that by using a collagenase-based isolation method, it is possible to successfully isolate primary cultures of hLECs without the use of 3T3s as a feeder layer. Chen and colleagues isolate clusters of limbal epithelium that contain supporting niche cells and separate these clusters from stromal cells before culturing. In our studies we digested only the superficial limbus and cultured the superficial stromal cells together with the limbal epithelial clusters and found that even though cultures were significantly improved in the presence of a 3T3 feeder layer, cultures of hLEC were also successful in SM without a 3T3 feeder layer. This provides further evidence that, with the right isolation technique and optimized cell culture media, it may be possible to isolate LECS for transplantation without the use of a 3T3 feeder layer. 
In summary, and to the best of our knowledge, this is the first study that has examined collagenase digestion of the superficial corneal limbus to isolate hLECs from OCCS rims. Due to the findings we present in this manuscript, we propose that surplus OCCS rims, which might have previously been disregarded, can now be used as a valuable source of research tissue for the isolation of hLECs and possibly LESCs. We also suggest a system whereby hLECs can be isolated without a 3T3 feeder layer by using a specific culture media and have highlighted a potential culture system in which CSSCs and hLECs can be isolated simultaneously from the same donor rim.

\section{ACKNOWLEDGEMENTS}

This study was supported by Shine the Light on Aniridia, The Special Trustees of Moorfields Eye Hospital and the National Institute for Health Research Biomedical Research Centre for Ophthalmology, Moorfields Eye Hospital and UCL Institute of Ophthalmology.

\section{REFERENCES}

1. Pels $\mathrm{E}$, Beele $\mathrm{H}$, Claerhout I. Eye bank issues: II. Preservation techniques: warm versus cold storage. Int Ophthalmol 2008;28:155-163.

2. Armitage WJ. Preservation of Human Cornea. Transfus Med Hemother 2011;38:143147.

3. Jones GL, Ponzin D, Pels E, Maas H, Tullo AB, Claerhout I. European eye bank association. Dev Ophthalmol 2009;43:15-21.

4. Shah K. Personal Communication. Eye Bank Manager of Moorfields Lions Eye Bank, London 2014.

5. Doughman DJ. Prolonged donor cornea preservation in organ culture: long-term clinical evaluation. Trans Am Ophthalmol Soc 1980;78:567-628.

6. Sperling S. Human corneal endothelium in organ culture. The influence of temperature and medium of incubation. Acta Ophthalmol (Copenh) 1979;57:269-276.

7. Sperling S. Endothelial cell density in donor corneas. Acta Ophthalmol (Copenh) 1980;58:278-282.

8. Joseph A, Powell-Richards AO, Shanmuganathan VA, Dua HS. Epithelial cell characteristics of cultured human limbal explants. Br J Ophthalmol 2004;88:393-398.

9. Zito-Abbad E, Borderie VM, Baudrimont $M$, et al. Corneal epithelial cultures generated from organ-cultured limbal tissue: factors influencing epithelial cell growth. Curr Eye Res 2006;31:391-399.

10. Borderie V, Borderie $\mathrm{P}$, Basli $\mathrm{E}$, et al. [Human limbal epithelial cell growth kinetics in vitro]. Journal francais d'ophtalmologie 2010;33:465-471.

11. Shanmuganathan VA, Rotchford AP, Tullo AB, Joseph A, Zambrano I, Dua HS. Epithelial proliferative potential of organ cultured corneoscleral rims; implications for allolimbal transplantation and eye banking. Br J Ophthalmol 2006;90:55-58.

12. Chen SY, Hayashida Y, Chen MY, Xie HT, Tseng SC. A new isolation method of human limbal progenitor cells by maintaining close association with their niche cells. Tissue Eng Part C Methods 2011;17:537-548.

13. Gonzalez S, Deng SX. Presence of native limbal stromal cells increases the expansion efficiency of limbal stem/progenitor cells in culture. Exp Eye Res 2013;116:169176.

14. Du Y, Funderburgh ML, Mann MM, SundarRaj N, Funderburgh JL. Multipotent stem cells in human corneal stroma. Stem Cells 2005;23:1266-1275. 
15. Kureshi AK, Funderburgh JL, Daniels JT. Human corneal stromal stem cells exhibit survival capacity following isolation from stored organ-culture corneas. Investigative ophthalmology \& visual science 2014;55:7583-7588.

16. Barrandon $\mathrm{Y}$, Green $\mathrm{H}$. Three clonal types of keratinocyte with different capacities for multiplication. Proc Natl Acad Sci U S A 1987;84:2302-2306.

17. Levis HJ, Menzel-Severing J, Drake RA, Daniels JT. Plastic compressed collagen constructs for ocular cell culture and transplantation: a new and improved technique of confined fluid loss. Curr Eye Res 2013;38:41-52.

18. Baylis O, Rooney P, Figueiredo F, Lako M, Ahmad S. An investigation of donor and culture parameters which influence epithelial outgrowths from cultured human cadaveric limbal explants. J Cell Physiol 2013;228:1025-1030.

19. James SE, Rowe A, llari L, Daya S, Martin R. The potential for eye bank limbal rings to generate cultured corneal epithelial allografts. Cornea 2001;20:488-494.

20. Koizumi N, Cooper LJ, Fullwood NJ, et al. An evaluation of cultivated corneal limbal epithelial cells, using cell-suspension culture. Investigative ophthalmology \& visual science 2002;43:2114-2121.

21. Zhang $X$, Sun $\mathrm{H}$, Tang $\mathrm{X}$, et al. Comparison of cell-suspension and explant culture of rabbit limbal epithelial cells. Exp Eye Res 2005;80:227-233.

22. Utheim TP, Raeder S, Utheim OA, et al. A novel method for preserving cultured limbal epithelial cells. Br J Ophthalmol 2007;91:797-800.

23. Jiang $\mathrm{Y}$, Jahagirdar BN, Reinhardt RL, et al. Pluripotency of mesenchymal stem cells derived from adult marrow. Nature 2002;418:41-49.

24. Loureiro RR, Cristovam PC, Martins CM, et al. Comparison of culture media for ex vivo cultivation of limbal epithelial progenitor cells. Mol Vis 2013;19:69-77.

25. Kruse FE, Tseng SC. Proliferative and differentiative response of corneal and limbal epithelium to extracellular calcium in serum-free clonal cultures. J Cell Physiol 1992;151:347360.

26. Lekhanont K, Choubtum L, Chuck RS, Sa-ngiampornpanit T, Chuckpaiwong V, Vongthongsri A. A serum- and feeder-free technique of culturing human corneal epithelial stem cells on amniotic membrane. Mol Vis 2009;15:1294-1302.

27. Mimura T, Yamagami S, Uchida S, et al. Isolation of adult progenitor cells with neuronal potential from rabbit corneal epithelial cells in serum- and feeder layer-free culture conditions. Mol Vis 2010;16:1712-1719.

28. Lu R, Bian F, Lin J, et al. Identification of human fibroblast cell lines as a feeder layer for human corneal epithelial regeneration. PLoS One 2012;7:e38825.

29. Scafetta G, Tricoli E, Siciliano C, et al. Suitability of human Tenon's fibroblasts as feeder cells for culturing human limbal epithelial stem cells. Stem Cell Rev 2013;9:847-857.

\section{FIGURE LEGENDS}

\section{Figure 1.}

Isolation of hLECs and CSSCs from OCCS rims. A) The superficial limbus was cut away from the remaining stroma and $B$ ) dissected into $2 \mathrm{~mm}$ pieces before overnight collagenase digestion. Scale Bar, 400 $\mu \mathrm{m}$. C) Evidence of limbal crypts could be seen in superficial segments under the dissecting microscope. Scale Bar, 100 $\mu \mathrm{m}$ D-E) Limbal epithelial clusters were visible after overnight collagenase digestion. F) Colonies of hLECs started to grow after 24 hours in culture. G-H) After 7 days in culture, epithelial colonies (black arrow) grew well

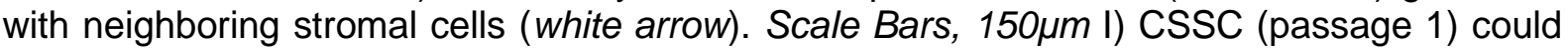
also be isolated from the same cultures in which hLECs were isolated. Scale Bar, $400 \mu m$ 


\section{Figure 2.}

Expression of pan-cytokeratins and vimentin in limbal epithelial clusters. A) Low resolution micrograph showing overall pan-cytokeratin (PCK, green) and vimentin (Vim, red) expression. Vimentin expression is evident throughout the cluster. B) High resolution micrographs showing Vim+/PCK- cells amongst PCK+/Vim- cells (arrows). C) Line scan zstack showing a cross section of a limbal epithelial cluster. Vim+/PCK- cells can be seen lying in a basal position to PCK+/Vim- limbal epithelial cells.

\section{Figure 3.}

Effects of different isolation techniques on hLEC culture were assessed. A) OCCS rims were bisected into nasal and temporal segments and subjected to either dispase or collagenase digestion. Cell suspensions were plated into 6 well plates and cultured for 7 days. The collagenase method of isolation was superior in terms of hLEC coverage, colony number and hLEC morphology. Scale bars, 160 $\mu \mathrm{m}$. B) OCCS rims were subjected to collagenase digestion, plated in $55 \mathrm{~mm} 2$ culture dishes and cultured for 14 days. Successful isolation (top) of hLECs using the collagenase method occurred in $80.55 \%$ of cultures and failure of hLEC isolation (bottom) occurred in $19.45 \%$ of cultures out of 36 OCCS rims.

\section{Figure 4.}

Effects of different cell media and 3T3 feeders on hLEC colony formation were investigated. Collagenase digestion of OCCS rims was carried out and cell suspensions were plated on $55 \mathrm{~mm} 2$ dishes. Representative photomicrographs of colonies at days 1 and 7 and photographs at day 14 were captured. Initial colonies formed at day 1 were similar in size and morphology with slightly smaller colonies present in cultures with 3T3 feeders. Scale bars, $325 \mu \mathrm{m}$. By day 7 , there was a noticeable difference in colony size. Cultures maintained in SM appeared to thrive more in comparison to cultures maintained in CM. Scale bars, $1755 \mu \mathrm{m}$. Similarly, on day 14 , epithelial colony size and coverage was noticeably higher in cultures maintained in SM compared to cultures maintained in CM.

\section{Figure 5.}

Isolation of hLECs from OCCS rims favors SM over CM and 3T3 feeders (F) over no feeders. A) Total hLEC colonies were counted across 8 different donors at day 7 of culture. Total colony count was significantly higher in cultures with 3T3 feeders compared to those without. The number of hLEC colonies was also significantly higher in SM cultures compared to CM cultures. B) Although there was no statistical difference in total colony number between $\mathrm{CM}(\mathrm{F})$ and $\mathrm{SM}(\mathrm{F})$, there was a significantly higher number of aborted colonies in $\mathrm{CM}(\mathrm{F})$ cultures compared to $\mathrm{SM}(\mathrm{F})$ cultures. C) In agreement with graph $\mathrm{B}$, there was also a significantly lower number of cells counted in $\mathrm{CM}(\mathrm{F})$ cultures compared to $\operatorname{SM}(F)$ cultures. Cell number was also significantly higher in culture maintained in SM compared to CM and in $\mathrm{CM}(\mathrm{F})$ compared to $\mathrm{CM}$. D) Percentage of successful isolations compared to failed isolations in 8 OCSCS rims. Isolations were considered a success if the colony number was greater than zero and a failure if colony number was zero. Data are mean \pm SEM for 8 OCCS rims, $\left({ }^{*} p<0.05 ;{ }^{* *} p<0.01\right.$; students unpaired t-test)

\section{Figure 6.}

SM produced epithelial cells with a higher cell density than CM. A) Cell density was calculated based on the higher the cell density, the smaller the cells. Following 7 days in culture, three photomicrographs of confluent patches of epithelial cells were captured per growth condition for each donor and all cells in each field of view were counted. SM cultures produced a significantly higher cells density than CM cultures. Data are mean \pm SEM for 8 
OCSCS rims ( ${ }^{* *} p<0.01$; students unpaired t-test). B) Representative photomicrographs

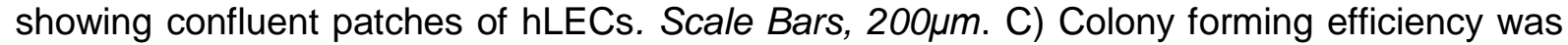
compared between different conditions and no significant differences were found between each. Data are mean \pm SEM for 5 OCCS rims. D) Images show representative colonies for each condition.

\section{Figure 7.}

Protein expression of hLECs isolated in SM and CM was investigated. Immunofluorescence confirmed universal expression of p63a in nuclei of hLECs and positive expression of the differentiation marker was only detected in a few large, more differentiated, suprabasal cells (arrows). Universal expression of the corneal epithelial marker pax6 was also detected in the nuclei of hLECs. No differences were observed between protein expression of hLECs

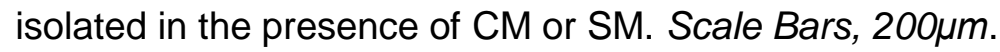

\section{Figure 8.}

Limbal epithelial cells displayed a more corneal like phenotype in 3D when maintained in SM. Epithelial cells were expanded in the presence of SM for 10 days before seeding onto RAFT TEs. RAFT TEs were maintained either in CM or SM for 7 days then airlifted for 7 days before fixing and staining. A) Representative confocal z-stack projections and cross sections (CS) show f-actin (red, Phalloidin) and nuclear (blue, DAPI) staining. Epithelial cells maintained in the presence of SM showed a more uniform corneal phenotype and were more likely to multilayer then cells grown in the presence of CM, which displayed larger cells with a less uniform morphology and a more differentiated phenotype. Scale Bars, 45 $\mu \mathrm{m}$. B) Immunofluorescence confirmed universal expression of p63 $\alpha$ and pax6 in nuclei of hLECs and expression of the differentiation marker CK3 was undetected. 